\title{
Estimation of non-statistical uncertainty using fuzzy-set theory
}

\author{
Xintao Xia $\uparrow$, Zhongyu Wang $\dagger$ and Yongsheng Gao $\ddagger$ \\ $\dagger$ Luoyang Institute of Technology, Luoyang 471 039, People's Republic of China \\ \$ Hong Kong University of Science and Technology, Clear Water Bay, Kowloon, Hong Kong \\ E-mail: mewan@lit.net.cn (Z Wang) and meygao@ust.hk (Y Gao)
}

Received 26 October 1999, in final form 18 January 2000, accepted for publication 3 February 2000

\begin{abstract}
A novel method using a fuzzy practicable interval to characterize non-statistical uncertainty in dynamic measurement is proposed. The method permits the uncertainty being estimated under the conditions that the number of measurements is very small and the probability distribution unknown. The feasibility of the method is validated by computer-simulation experiments.
\end{abstract}

Keywords: non-statistical uncertainty, estimation, dynamic measurement, fuzzy-set theory

\section{Introduction}

Measurement uncertainty shows the range within which the true value of a measurand is to be estimated [1]. It consists of statistical and non-statistical uncertainty [2-4]. For statistical uncertainty, some models have already been established [5]. If the probability-distribution density $p(x)$ of measured values obeys a normal distribution, its uncertainty can be estimated as $k \sigma_{B}$, where $k$ is the confidence coefficient and $\sigma_{B}$ is the standard deviation obtained by the Bessel method. If $p(x)$ is a non-normal distribution, a considerable estimation error is likely to occur if the same method is used $[1,4,5]$.

The problem of non-normal distribution of measured values has been studied by several authors. Some typical distributions were examined by Sachs [6] using the squaregraph method and the probability-paper method. Manonkian [7] and Pugachev [8] used the range-enlargement method. Hart [9] investigated the probability-treatment method.

Kubisa and Turzeniecka [10] evaluated a number of methods for approximating uncertainty in measurement when the convolution of the probability distribution of the error component as well as the output confidence level were known. Shu [11] suggests that the $\beta$ distribution of the parameters $\alpha$ and $\beta$ should be used for approximating the sample distribution; the confidence coefficients $k_{j}$ and $k_{a}$ can thus be obtained. The conceptual uncertainty [12] reveals three different ensembles of random values with distinct distribution laws. Bayesian theory and the maximumentropy principle $[13,14]$ are based on statistics or the probability density.

The non-parametric statistical method [15] is of interest for non-Gaussian data under certain conditions. The method was further investigated by Lasserre et al [16] and Qin and Wang [17] in order to develop supplementary materials for GUM (Guide to the Expression of Uncertainty in Measurement) [3].

In dynamic measurements, estimation of the nonstatistical uncertainty is very important, because not all the uncertainty can be estimated with the statistical method [18]. Furthermore, the case is typically far more complex than the statistical uncertainty. For example, in rocket-firing experiments and some destructive experiments, the number of measured values is rather small and the probabilitydistribution density of experiments of this kind might be unknown. In these cases, treating the problem by the available methods would be rather difficult.

To solve the above problems, the authors developed a new method using a fuzzy practicable interval to express the non-statistical uncertainty in dynamic measurement. The method allows the number of measurement values to be very small and the probability distribution unknown.

\section{The fuzzy interval of measured data}

By use of subordination functions, fuzzy mathematics [19] researches transition laws of a fuzzy entity changing from true tofalse or from false to true. In the measurement, the true value $X_{0}$ always exists uniquely and objectively. Therefore, we define a set $A$ as

$$
A=X_{0}
$$

The set $A$ contains a single value $X_{0}$.

In set theory, for the measured values $x_{i}, i=1,2, \ldots, n$, the set $A$ has the following characteristic function:

$$
G_{A}(x)= \begin{cases}1 & x_{i} \in A \\ 0 & x_{i} \notin A\end{cases}
$$

where 1 represents true and 0 represents false. $n$ is the number of measurements or the number of measurement samples. 


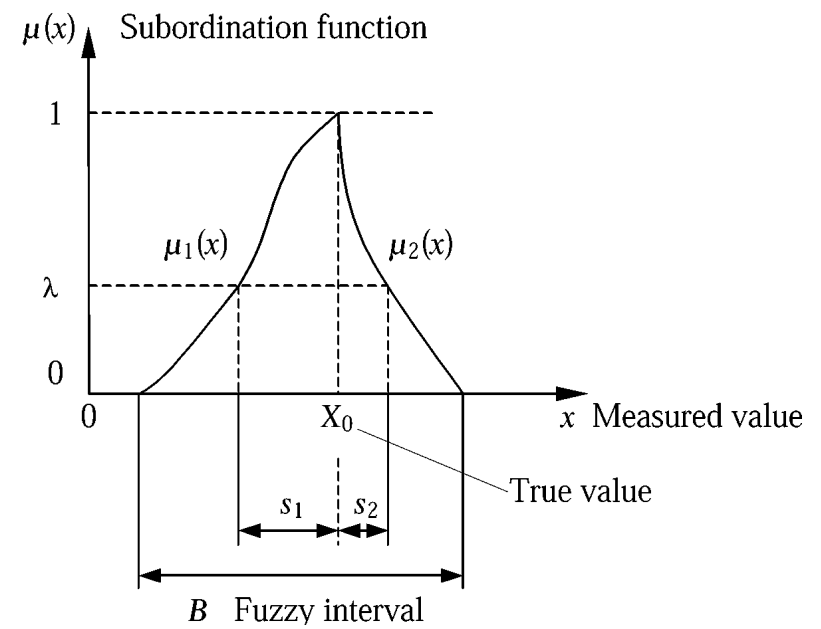

Figure 1. The subordination function and measured values.

In fuzzy-set theory, a transition can be considered to exist in the subordination of $x_{i}$ in relation to $A$ and the interval $B$ of the transition can be described by the subordination function (figure 1):

$$
\mu(x)= \begin{cases}\mu_{1}(x) & x_{i} \leqslant X_{0} \\ \mu_{2}(x) & x_{i} \geqslant X_{0}\end{cases}
$$

where $\mu_{1}(x) \in[0,1]$ and $\mu_{2}(x) \in[0,1]$. The function $\mu(x)$ describes how the measured value $x_{i}$ accords with the set $A$. From figure 1, it can be seen that $\mu_{1}(x)$ increases while $\mu_{2}(x)$ decreases. $\lambda \in[0,1]$. Two ranges $s_{1}$ and $s_{2}$ near $X_{0}$ on the $x$ axis can be found. The interval of $x$ subordinate to the set $A$ is

$$
U_{F_{\lambda}}=s_{1}+s_{2}
$$

where $\mu_{A_{\lambda}}=\lambda$. The value of $\mu(x)$ is the degree of subordination of $x$ to the set $A$. In the measured value $x_{i}$, given $\lambda=\lambda^{*}$, then $U_{F_{\lambda}}=U_{F_{\lambda} *}$ is uniquely determined. This shows that the dispersal range of the measured value $x_{i}$ is $U_{F_{\lambda} *}$ relative to the true value $X_{0}$ (figure 1). In figure 1, $B$ is the fuzzy interval, $\lambda^{*}$ is the optimum level and $U_{F_{2} *}$ is the fuzzy practicable interval under the $\lambda^{*}$ level. Then the characteristic function is given as

$$
G_{A_{\lambda}}(x)= \begin{cases}1 \text { (true) } & \mu_{A}(x) \geqslant \lambda^{*} \\ 0 \text { (false) } & \mu_{A}(x)<\lambda^{*} .\end{cases}
$$

Equation (5) shows that values of $x$ in the interval $U_{F_{\lambda *}}$ are usable, to be represented by 1 (true), whereas those outside the interval $U_{F_{\lambda^{*}}}$ are unusable, to be represented by 0 (false). On the basis of measurement theory, the uncertainty in measurement can be identified by $U_{F_{\lambda^{*}}}$.

\section{The determination of $\lambda^{*}$ and $X_{0}$}

In terms of fuzzy-set theory, $\lambda^{*}$ determines an entity's border from one extreme to another. In fact, $\lambda^{*}$ can also be regarded as a fuzzy number and its fuzzy character reaches the peak when its value equals 0.5 , both true and false. $\lambda \geqslant 0.5$ means that the most usable $x$ is included in the set $A$. Therefore, in theory, $\lambda^{*}$ can be determined to be 0.5 . In practical data
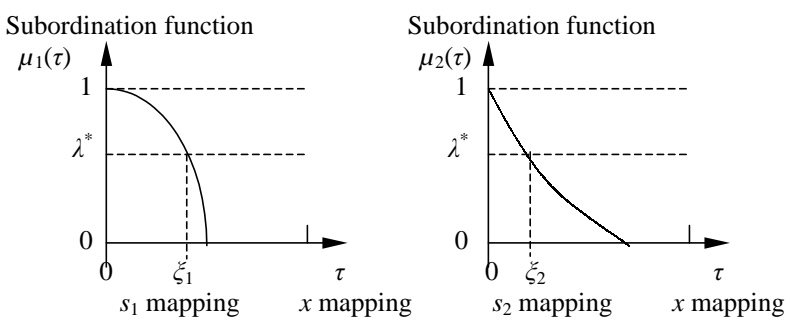

Figure 2. Subordination functions and mapping parameters.

treatment, $\lambda^{*}=0.4-0.5$ in general. If $n$ is rather small, for example $n \leqslant 200, \lambda^{*}=0.4$ can be adopted.

Generally speaking, the true value $X_{0}$ is unknown and it could be estimated by statistical analysis [20]. In figure 1 , $x_{v}$, the value of $x$ when $\mu(x)=1$, is used to estimate $X_{0}$ as

$$
\left.X_{0} \approx x\right|_{\mu(x)=1}=x_{v} .
$$

\section{Parameter mapping}

The subordination function in fuzzy mathematics can be identified by a probability-distribution-density function in error theory. If $p=p(x)$ is known, the linear transformation

$$
\mu(x)=\left(p(x)-p_{\min }\right) /\left(p_{\max }-p_{\min }\right) \quad p_{\min } \neq p_{\max }
$$

maps $p$ into the interval $[0,1]$ and hence $\mu(x)$ can be obtained. In equation (7), the suffix $\min$ and the suffix $\max$ stand for the minimum and maximum values, respectively. Equations (6) and (7) conclude that $x_{v}$ is in correspondence with $p_{\max }$, which is the maximum value of the probabilitydistribution-density function.

Since $x_{i}$ is already regarded as a fuzzy number, it lies in the interval $[0,1]$. Therefore, the linear transformations

$$
\begin{gathered}
\eta_{v}=\left(x_{v}-x_{\text {min }}\right) /\left(x_{\text {max }}-x_{\text {min }}\right) \\
\eta(x)=\left(x-x_{\text {min }}\right) /\left(x_{\text {max }}-x_{\text {min }}\right) \\
\tau=\tau(x)=\left|\eta(x)-\eta_{v}\right|=\left|x-x_{v}\right| /\left(x_{\text {max }}-x_{\text {min }}\right)
\end{gathered}
$$

map $x$ into the interval $[0,1]$ and the measured value written as the fuzzy number $\tau(x)$ is obtained, where $x_{v}$ can be expressed as $\tau_{v}=0$.

In the interval $[0,1], \psi_{F_{\lambda}}$ stands for $U_{F_{\lambda}}$ and $\xi_{1}$ and $\xi_{2}$ stand for $s_{1}$ and $s_{2}$, respectively. Then equation (4) can be written as

$$
\begin{aligned}
& U_{F_{\lambda}}=s_{1}+s_{2}=\left(\left|x-x_{v}\right|_{\mu_{1}(x)=\lambda}\right)+\left(\left|x-x_{v}\right|_{\mu_{2}(x)=\lambda}\right) \\
& \quad=\left(x_{\max }-x_{\min }\right)\left(\left.\tau\right|_{\mu_{1}(\tau)=\lambda}\right)+\left(x_{\max }-x_{\min }\right)\left(\left.\tau\right|_{\mu_{2}(\tau)=\lambda}\right) \\
& \quad=\left(\left.\tau\right|_{\mu_{1}(\tau)=\lambda}+\left.\tau\right|_{\mu_{2}(\tau)=\lambda}\right)\left(x_{\max }-x_{\min }\right) \\
& =\left(\xi_{1}+\xi_{2}\right)\left(x_{\max }-x_{\min }\right)=\psi_{F_{\lambda}}\left(x_{\max }-x_{\min }\right) \\
& \psi_{F_{\lambda}}=\xi_{1}+\xi_{2} .
\end{aligned}
$$

On the basis of the above, figure 1 can be extended further, as shown in figure 2 .

If the discrete values $\mu_{1 j}\left(\tau_{j}\right)$ and $\mu_{2 j}\left(\tau_{j}\right), j=1,2, \ldots$, are known, $\mu_{1}(\tau)$ and $\mu_{2}(\tau)$ can be obtained using the following method. Define the maximum norm

$$
\|r\|_{\infty}=\max \left|r_{j}\right| \quad j=1,2, \ldots
$$


Use the polynomials

$$
\begin{aligned}
& f_{1}=f_{1}(\tau)=1+\sum_{l=1}^{L} a_{l} \tau^{l} \\
& f_{2}=f_{2}(\tau)=1+\sum_{l=1}^{L} b_{l} \tau^{l}
\end{aligned}
$$

to approximate discrete values $\mu_{1 j}\left(\tau_{j}\right)$ and $\mu_{2 j}\left(\tau_{j}\right)$, respectively. We obtain

$$
\begin{aligned}
& \mu_{1}(\tau)=f_{1}(\tau) \\
& \mu_{2}(\tau)=f_{2}(\tau) .
\end{aligned}
$$

Suppose that

$$
\begin{gathered}
r_{1 j}=f_{1}\left(\tau_{j}\right)-\mu_{1 j}\left(\tau_{j}\right) \quad j=1,2, \ldots, v \\
r_{2 j}=f_{2}\left(\tau_{j}\right)-\mu_{2 j}\left(\tau_{j}\right) \quad j=v, v+1, \ldots
\end{gathered}
$$

Select $a_{l}=a_{l}^{*}$ that satisfies

$$
\min \left\|r_{1}\right\|_{\infty}
$$

Select $b_{l}=b_{l}^{*}$ that satisfies

$$
\min \left\|r_{2}\right\|_{\infty}
$$

Then the unknown coefficients $a_{l}$ and $b_{l}$ can be obtained. In equations (14) and (15), $L$, the degree of polynomials, is typically equal to 3 or 4 .

The restraint conditions in equations (20) and (21) can be expressed as

$$
\begin{aligned}
& f_{1}^{\prime}=\mathrm{d} f_{1} / \mathrm{d} \tau \leqslant 0 \\
& f_{2}^{\prime}=\mathrm{d} f_{2} / \mathrm{d} \tau \leqslant 0 .
\end{aligned}
$$

This reveals a steadily decreasing characteristic regarding the subordination functions. This method is called the maximum-norm method and the error of the approximation is less than that of the least-squares method. $\xi_{1}$ and $\xi_{2}$ can be obtained from the following expressions:

$$
\begin{aligned}
& \min \left|\mu_{1}(\tau)-\lambda^{*}\right|_{\tau=\xi_{1}} \\
& \min \left|\mu_{2}(\tau)-\lambda^{*}\right|_{\tau=\xi_{2}} .
\end{aligned}
$$

The right-square-graph estimation method is described first. When $n$ is rather large, for example $n \geqslant 30$, the measured values can be divided into $q$ groups. In each group, the median is $d_{j}$ and the frequency is $m_{j}$.

Let the median of the group having the largest frequency be $x_{v}$ and the number of the group be $v$. If there are $t$ repeated frequencies, $x_{v}$ and $v$ can be determined by the mean method. Then

$$
\begin{gathered}
p_{1 j}\left(x_{j}\right)=m_{j} \quad j=1,2, \ldots, v \\
p_{2 j}\left(x_{j}\right)=m_{j} \quad j=v, v+1, \ldots, q+1 .
\end{gathered}
$$

Another approach is to use the linear estimation method. Arrange $x_{i}$ from small to large to form a new order as

$$
x_{i}^{*} \leqslant x_{i+1}^{*} \quad i=1,2, \ldots, n-1 .
$$

Define

$$
\Delta_{i}=x_{i+1}^{*}-x^{*} \geqslant 0
$$

In general, the smaller $\Delta_{i}$ the thicker the distribution of the measured values. Conversely, it will be thinner. $\Delta_{i}$ and $x_{i}$ are related to the distribution density. We use the linear functions

$$
\begin{gathered}
m_{j}=1-\left(\Delta_{j}-\Delta_{\min }\right) / \Delta_{\max } \quad \Delta_{\max }=\max \Delta_{j} \\
\Delta_{\min }=\min \Delta_{j} \quad j=1,2, \ldots, n-1
\end{gathered}
$$

to approximate the probability-distribution-density function. Similarly to the right-square-graph estimation method, $x_{v}$ and $v$ can be obtained. Therefore,

$$
\begin{gathered}
p_{1 j}\left(x_{j}^{*}\right)=m_{j} \quad j=1,2, \ldots, v \\
p_{2 j}\left(x_{j}^{*}\right)=m_{j} \quad j=v, v+1, \ldots, n .
\end{gathered}
$$

$\mu_{1 j}\left(\tau_{j}\right)$ and $\mu_{2 j}\left(\tau_{j}\right)$ can be obtained using equations (7)-(23).

\section{The estimation algorithm}

The algorithm of the method is based on the optimal theory. The target functions are equations (20), (21), (24) and (25). The constraint conditions are equations (22) and (23). The optimal method used is SUMT [21]. The algorithm is summarized as follows.

(i) Sample $x_{i} \quad i=1,2, \ldots, n$.

(ii) Obtain a new order $x_{i}^{*}$ according to equation (28).

(iii) Compute $p_{1 j}\left(x_{j}^{*}\right)=m_{j}(j=1,2, \ldots, v)$ and $p_{2 j}\left(x_{j}^{*}\right)=m_{j}(j=v, v+1, \ldots, n)$ according to equations (29)-(32) after obtaining $v$ and $x_{v}$.

(iv) Compute $\eta(x)$ and $\tau$ according to equations (9) and (10) after obtaining $\mu_{1 j}\left(\tau_{j}\right)(j=1,2, \ldots, v)$ and $\mu_{2 j}\left(\tau_{j}\right)$ $(j=v, v+1, \ldots, n)$ from equation (7).

(v) Establish the models for $f_{1}$ and $f_{2}$ from equations (14), (15) and (18)-(21) under the condition of equations (22) and (23).

(vi) Obtain the subordination functions $\mu_{1}(\tau)$ and $\mu_{2}(\tau)$ according to equations (16) and (17).

(vii) Obtain $U_{F_{\lambda^{*}}}$ according to equation (11) after computing $\xi_{1}$ and $\xi_{2}$ under the level $\lambda=\lambda^{*}$ from equations (24) and (25).

\section{Case studies}

The estimation error of the proposed method is to be examined. The simulated data are used to analyse the uncertainty in the estimation error and the normal distribution, Rayleigh distribution, triangle distribution and uniform distribution are considered in the investigation.

The simulated values $x_{i}$ of the four distributions are generated by the computational method. The values of $U_{F_{\lambda^{*}}}$ for the four distributions are obtained. Suppose that the true value of the uncertainty is $U$; then the relative error in the estimation for $U$ can be defined as

$$
\Delta U=\left|U_{F_{\lambda^{*}}}-U\right| / U
$$

In the simulation, $\lambda^{*}=0.4$ to take a small sample $(4 \leqslant n \leqslant$ 50). $\mu(x)$ can be estimated using the proposed estimation method. 
Table 1. Results of the simulation and case studies $(n=6-10)$.

\begin{tabular}{llcccc}
\hline & \multicolumn{4}{c}{ Distribution } \\
\cline { 3 - 6 } & Normal & Rayleigh & Triangle & Uniform \\
\hline True value of uncertainty & 0.6 & 1.72301 & 1.0 & 1.0 \\
Proposed & Estimated & 0.574453 & 1.842456 & 1.069633 & 0.95879 \\
method & uncertainty & 0.333788 & & 0.924679 & \\
& Relative error (\%) & 4.26 & 6.932 & 6.96 & 4.121 \\
& & 44.37 & & 7.53 & \\
Bessel & Estimated & 0.69054 & 2.09777 & 1.22555 & 1.77381 \\
method & uncertainty & 0.37594 & & 1.41687 & \\
& Relative error (\%) & 15.09 & 21.75 & 22.56 & 77.38 \\
& & 37.34 & & 41.69 & \\
\hline
\end{tabular}

Table 2. Results of the simulation and case studies $(n=4)$.

\begin{tabular}{|c|c|c|c|c|c|}
\hline & & \multicolumn{4}{|c|}{ Distribution } \\
\hline & & Normal & Rayleigh & Triangle & Uniform \\
\hline \multicolumn{2}{|c|}{ True value of uncertainty } & 0.6 & 1.72301 & 1.0 & 1.0 \\
\hline \multirow[t]{2}{*}{$\begin{array}{l}\text { Proposed } \\
\text { method }\end{array}$} & $\begin{array}{l}\text { Estimated } \\
\text { uncertainty }\end{array}$ & 0.48001 & 1.41093 & 0.94380 & 0.82955 \\
\hline & Relative error $(\%)$ & 20 & 18.11 & 5.62 & 17.05 \\
\hline
\end{tabular}

\subsection{The normal distribution}

The first set of simulated values $x_{i}(n=10)$ is as follows:

$\begin{array}{lllll}50.02170 & 50.04282 & 50.06641 & 49.92250 & 49.71110 \\ 50.00218 & 50.12576 & 50.03276 & 50.02137 & 49.91243 .\end{array}$

The true value $U$ in the simulation is $U=6 \sigma=0.6$.

The uncertainty estimated using the proposed method is $U_{F_{\lambda^{*}}}=U_{F_{0.4}}=0.574453$. The relative error of the estimation is

$$
\Delta U=|0.574453-0.6| / 0.6=4.26 \% .
$$

By Bessel estimation, the uncertainty is $6 \sigma_{B}=0.69054$. The relative error of estimation is $15.09 \%$.

The second set of simulated values $x_{i}(n=10)$ is as follows:

$$
\begin{array}{lllll}
49.93576 & 49.99186 & 49.98047 & 49.97154 & 49.99512 \\
49.85121 & 49.93983 & 49.96095 & 50.08453 & 50.04063 .
\end{array}
$$

The true value $U$ in the simulation is $U=6 \sigma=0.6$.

The uncertainty estimated using the proposed method is $U_{F_{*} *}=U_{F_{0.4}}=0.333788$. The relative error of the estimation is $44.37 \%$.

By the Bessel estimation, the uncertainty is $6 \sigma_{B}=$ 0.37594 . The relative error of the estimation is $37.34 \%$.

\subsection{The Rayleigh distribution}

The simulated values $x_{i}(n=10)$ are as follows:

$$
\begin{array}{lllll}
1.6201 & 2.138 & 0.8592 & 1.54193 & 1.20955 \\
1.74325 & 1.18994 & 1.52684 & 1.522 & 1.65953 .
\end{array}
$$

The true value $U$ in the simulation is $U=2 \times 2.636 \sigma_{R}=$ 1.72301
The uncertainty estimated using the proposed method is 1.842456 and the relative error of the estimation is only $6.932 \%$.

By the Bessel estimation, the uncertainty is $6 \sigma_{B}=$ 2.09777 and the relative error of the estimation is $21.75 \%$.

\subsection{The triangle distribution}

The first set of simulated values $x_{i}(n=10)$ is as follows:

$$
\begin{array}{lllll}
5.54424 & 5.625905 & 5.7059 & 5.691365 & 5.801535 \\
5.45576 & 5.374095 & 5.2941 & 5.308635 & 5.198465 .
\end{array}
$$

The interval of the simulation is $[5,6]$. The true value of the uncertainty is $U=6-5=1$.

The uncertainty estimated using the proposed method is 1.069633 and the relative error of the estimation is $6.96 \%$.

By the Bessel estimation, the uncertainty is $6 \sigma_{B}=$ 1.22555 and the relative error of the estimation is $22.56 \%$.

The second set of simulated values $x_{i}(n=8)$ is as follows:

$$
\begin{array}{llll}
5.56117 & 5.59951 & 5.920055 & 5.58392 \\
5.4883 & 5.40049 & 5.079945 & 5.41608 .
\end{array}
$$

The interval is $[5,6]$ and the true value of the uncertainty is $U=6-5=1$.

The uncertainty estimated using the proposed method is 0.924679 and the relative error of the estimation is $7.53 \%$.

By the Bessel estimation, the uncertainty is $6 \sigma_{B}=$ 1.41687 and the relative error of the estimation is $41.69 \%$.

\subsection{The uniform distribution}

The simulated values $x_{i}(n=7)$ are as follows:

$$
\begin{array}{lll}
5.050303 & 5.57313 & 5.33894
\end{array}
$$

$\begin{array}{llll}5.00067 & 5.3255 & 5.85851 & 5.42444 .\end{array}$ 
Table 3. Results of the simulation and case studies $(n=20)$.

\begin{tabular}{|c|c|c|c|c|c|}
\hline & & \multicolumn{4}{|c|}{ Distribution } \\
\hline & & Normal & Rayleigh & Triangle & Uniform \\
\hline \multicolumn{2}{|c|}{ True value of uncertainty } & 0.6 & 1.72301 & 1.0 & 1.0 \\
\hline \multirow[t]{2}{*}{$\begin{array}{l}\text { Proposed } \\
\text { method }\end{array}$} & $\begin{array}{l}\text { Estimated } \\
\text { uncertainty }\end{array}$ & 0.657 & 1.69218 & 0.92221 & 0.96604 \\
\hline & Relative error (\%) & 9.5 & 1.789 & 7.779 & 3.396 \\
\hline
\end{tabular}

Table 4. Results of the simulation and case studies $(n=50)$.

\begin{tabular}{llllll}
\hline & \multicolumn{4}{c}{ Distribution } \\
\cline { 3 - 6 } & Normal & Rayleigh & Triangle & Uniform \\
\hline True value of uncertainty & 0.6 & 1.72301 & 1.0 & 1.0 \\
$\begin{array}{l}\text { Proposed } \\
\text { method }\end{array}$ & $\begin{array}{l}\text { Estimated } \\
\text { uncertainty }\end{array}$ & 0.626 & 1.81250 & 1.10173 & 0.932663 \\
& Relative error $(\%)$ & 4.333 & 5.194 & 10.173 & 6.734 \\
\hline
\end{tabular}

The interval is $[5,6]$ and the true value of the uncertainty is $U=6-5=1$.

The uncertainty estimated using the proposed method is 0.95879 and the relative error of the estimation is $4.121 \%$.

By the Bessel estimation, the uncertainty is $6 \sigma_{B}=$ 1.77381 and the relative error of the estimation is $77.38 \%$.

\subsection{Summary}

The results of the above four cases are summarized in table 1 . To further demonstrate the effectiveness of the proposed method, the sampling number $n$ is varied for the above cases and the results are shown in table 2-4.

\section{Discussion}

It can be seen that the proposed method can be used for the normal, Rayleigh, triangle and uniform distributions both for large $n$ and for small $n$. According to the results in tables 14 , the proposed method can be used for small samples, for example as few as four elements. The results for samples of six or more elements are rather good.

The simulation results show that, when measured values conform to the normal distribution, the relative error of the estimation using the proposed method is very small. The confidence level has been computed and it is up to $99.73 \%$. The relative error of the estimation using the Bessel method is very large under the condition of a small sampling number.

When measured values conform to non-normal distributions, such as the Rayleigh distribution, the triangle distribution and the uniform distribution, the results obtained using the proposed method are near to the true values of the uncertainty and the relative errors of the estimation are very small. However, using the Bessel method, the errors are fairly large. The main reason for the large error is that the Bessel method can be applied only under the condition of the distribution being normal.

This shows that the proposed method can be applied to various conditions, in particular, the conditions of nonnormal distributions. Thus, when the distributions of measured values are unknown, results that are very near to the true values can be obtained using the proposed method. The reason is that the estimation method developed can automatically recognize the subordination function $\mu(x)$ according to the discrete characteristics, which may be unknown prior to the measurement.

The uncertainty under $\lambda^{*}$ of measured values can be directly obtained using this method. With the new method, it is no longer necessary to estimate the standard deviation and the confidence coefficients. For systems of small samples and unknown distributions, the proposed method is more suitable.

\section{Conclusions}

The fuzzy practicable interval $U_{F_{\lambda}}$ can be used as the estimation parameter for the uncertainty of measured values. Its optimal level is $0.4-0.5 . U_{F_{\lambda}}$ is relative to $x_{v}$, the value of $x$ when the degree of subordination equals 1 . Therefore $x_{v}$ can be regarded as an estimation parameter of the true value.

The subordination function can be worked out from measured values through the right-square graph and the proposed estimation method without the need to know the probabilitydistribution density. It can be expressed as a polynomial under the condition of minimizing the maximum norm.

Using the fuzzy practicable interval $U_{F_{\lambda}}$ to estimate the uncertainty of measured values is characterized by allowing the distribution of measured values to be unknown and the number of samples to be very small. The proposed method can be applied to the estimation of non-statistical uncertainty in dynamic measurement.

\section{Acknowledgment}

This project is supported by the National Science Foundation of the People's Republic of China (grant 59805 007).

\section{References}

[1] Vujevic D 1994 Uncertainty of Measurement Automatika 35 35-9

[2] Bureau International des Poids et Mesures (BIPM) 1980 Expression of Experimental Uncertainties, Recommendation INC-1 
[3] ISO 1993 Guide to the Expression of Uncertainty in Measurement (Geneva: ISO)

[4] Michelini R C and Rossi G B 1995 Measurement uncertainty, a probabilistic theory for intensive entities Measurement 15 143-57

[5] Dieck R H 1997 Measurement uncertainty models ISA Trans. 36 29-35

[6] Sachs L 1982 Applied Statistics, A Handbook of Techniques (Berlin: Springer)

[7] Manonkian E B 1986 Modern Concepts and Theorems of Mathematics (Berlin: Springer)

[8] Pugachev V S 1984 Probability Theory and Mathematical Statistics for Engineers (Oxford: Pergamon)

[9] Hart H 1980 Non-Gaussian error boundaries Measurement for Progress in Science and Technology (Amsterdam: North-Holland)

[10] Kubisa S and Turzeniecka D 1996 Evaluation of some approximated methods of measurement uncertainty estimation Proc. Third Int. Symp. on Methods and Models in Automation and Robotics vol 2, pp 537-42

[11] Shu D 1991 Determination of the confidence coefficients $k_{i}$ and $k_{a}$ with regard to the structure of uncertainty, measurement errors Proc. 37th Int. Instrumentation Symp. (ISA) pp 107-13

[12] Sobolev V I 1996 Conceptual uncertainty of measurement errors Izmeritel'naya Tekhn. 39 7-8
[13] Weise K and Woger W 1993 A Bayesian theory of measurement uncertainty Meas. Sci. Technol. 4 1-11

[14] West B J 1987 Measurement, information and uncertainty Math. Computers Simulation 29 169-89

[15] Cox M G, Harris P M, Milton M J T and Woods P T 1997 A method for evaluating trends in ozone-concentration data and its application to data from the UK rural ozone monitoring Advanced Mathematical Tools in Metrology vol III, ed P Ciarlini et al (Singapore: World Scientific) pp 171-8

[16] Lasserre V, Mauris G and Foulloy L 1998 Fuzzy modeling of measurements acquired by an intelligent ultrasonic telemeter Instrumentation and Measurement Technology Conf. pp 837-42

[17] Qin P and Wang Z 1999 On non-statistic uncertainty in dynamic measurement Proc. 1st Int. Symp. on Instrumentation Science and Technology pp 228-32

[18] Liu Z 1995 Uncertainty and reliability analysis Proc. Quality Standardization Metrology

[19] Dubois D and Prade H 1980 Fuzzy Sets and Systems, Theory and Applications (New York: Academic)

[20] Jaech J L 1985 Statistical Analysis of Measurement Errors (New York: Wiley)

[21] Sun J and Mi C 1985 Optimal Design of Mechanical Structures (Harbin: Harbin Institute of Technology Press) 